\title{
MEASUREMENTS OF EXPONENTIAL GAIN AND SATURATION OF SASE AT THE APS LEUTL
}

\author{
S.V. Milton reporting for the APS LEUTL Commissioning Team \\ Argonne National Laboratory, 9700 South Cass Ave., Argonne, IL 60439 USA
}

\begin{abstract}
The possibility of using self-amplified spontaneous emission (SASE) to achieve a peak x-ray brightness nearly 11 orders of magnitude greater than available at today's third-generation light sources has in recent years generated tremendous excitement in the synchrotron light source community. With continued refinement in electron beam sources, electron beam control and metrology, and undulator magnet technology, this dream is rapidly approaching reality. The Advanced Photon Source (APS) Low-Energy Undulator Test Line (LEUTL) Free-Electron Laser (FEL) project is designed to explore the SASE process in the visible through vacuum ultraviolet spectral range, the current limit of the technology. At present we have made measurements of the SASE process at $530 \mathrm{~nm}$ and $385 \mathrm{~nm}$. We will present these measurements, in particular the growth of quantities such as intensity, spectrum, and microbunching along the length of the undulator. Measurements of these properties at and beyond the saturation point will also be presented. The experimental measurements will then be compared with theory and simulation.
\end{abstract}

\section{INTRODUCTION}

The progress of synchrotron radiation light sources is best observed by plotting on a semi-logarithmic scale the brilliance (the number of photons per second focused onto some area and contained within a specified solid angle and energy bandwidth) of available $\mathrm{x}$-ray sources as a function of time. It is immediately evident that this plot follows something similar to "Moore's Law." The brilliance of sources is seen to increase by roughly a factor of ten every three years. This is an astounding trend and a rate that surpasses even the semiconductor industry.

The present generation of machines, the so-called thirdgeneration synchrotron light sources, still have the ability to increase their brilliance. This will be done by exploiting lower emittance lattices, top-up operation, higher total currents, and longer and smaller gap undulators. But given enough time, these machines will not be able to keep pace with the historic trend.

There does exist, however, another branch to the evolutionary tree of synchrotron radiation sources--the free-electron laser. What sets the free-electron laser apart from the third-generation light sources is that the electrons within a bunch emit light coherently. In a thirdgeneration source the emission process of each electron is completely independent of its neighbors. A great deal can be gained if one can create a coherent emission of the electrons, as there is typically $>10^{9}$ electrons within a bunch. The resultant brilliance of such a source can be many orders of magnitude higher than present sources.

As an electron passes through an undulator magnet it emits synchrotron radiation in a relatively narrow bandwidth centered at the resonance frequency [1]

$$
\lambda_{r}=\frac{\lambda_{\text {und }}}{2 \gamma^{2}}\left(1+K^{2} / 2\right),
$$

where $\lambda_{\text {und }}$ is the undulator magnetic field period, $\gamma$ is the electron total energy normalized to its rest mass energy, and $K$ is the ratio of the maximum angle of the electron's sinusoidal trajectory within the undulator field, $\theta_{\max }$, to the radiation opening angle, $1 / \gamma$.

In a free-electron laser this EM field at the resonant frequency appears as a ponderomotive potential to the electrons. This potential traps the electrons and microbunches the beam at the resonant frequency. Electrons within a microbunch now act together and emit in phase. The electric field thus produced is linearly proportional to the number of electrons, and the intensity is proportional to the square of the number of electrons within the bunch. The difficulty lies in creating an initial ponderomotive potential that has sufficient strength to begin the microbunching.

In the basic FEL configuration mirrors are used at either end of the undulator to create an optical cavity. If the losses in the cavity are sufficiently small and the gain per pass of the optical field is large enough, then the intracavity optical power can grow to a size sufficiently large to develop regions that have large ponderomotive potentials on subsequent passages of bunches. Microbunching begins and the system power increases exponentially until the losses equal the gain per pass. This method relies on mirrors and so is typically limited to wavelengths in the UV to VUV range.

There are, however, other conditions that must be met for the FEL process to function. The emittance of the electron beam must be sufficiently small so that the beam size and divergence is not significantly larger than the $\mathrm{TEM}_{00}$ optical mode. The Rayleigh length must be long enough that there is time for the light to interact with the electron beam, and the energy spread in the beam must not be significantly larger than the relative bandwidth of the FEL process. The better one can meet these conditions the higher the single-pass gain and the simpler it is to achieve lasing.

If the electron beam quality is sufficient, the gain can be so large that the exponential process can begin in a single pass starting from the spontaneous emission of the electron bunch within the undulator. If the undulator is of sufficient length then the optical signal will grow 
exponentially to saturation. This process is known as selfamplified spontaneous emission (SASE). SASE requires no mirrors and so is scalable down to $\mathrm{x}$-ray wavelengths.

The relevant beam parameter for the FEL process is

$$
\begin{aligned}
& \rho=\left[\frac{I}{I_{A}} \frac{\gamma \lambda_{r}^{2}}{16 \pi^{2} \sigma_{\text {trans }}^{2}\left(1+K^{2} / 2\right)^{2}}\right]^{1 / 3} \\
& {\left[J_{0}\left(\frac{K^{2}}{4+2 K^{2}}\right)-J_{1}\left(\frac{K^{2}}{4+2 K^{2}}\right)\right]^{2 / 3},}
\end{aligned}
$$

where $I$ is the peak current of the electron bunch, $I_{A}=17,045 \mathrm{~A}$ is the Alfven current, $J_{0,1}$ are Bessel functions, $\sigma_{\text {trans }}$ is the electron bunch transverse rms size, $K=e B_{o} \lambda_{u} / 2 \pi m c^{2}$ is the dimensionless undulator strength parameter, $B_{o}$ is the maximum on-axis magnetic field strength of the undulator, and $\lambda_{u}$ is the undulator period. The gain length is the distance over which the power increases by a factor of e $(\sim 2.72)$. Under ideal conditions the one-dimensional gain length is given by

$$
L_{G}^{0}=\frac{\lambda_{u}}{4 \pi \sqrt{3} \rho} .
$$

Clearly one wishes to make $\rho$ as large as possible to reduce the gain length. This is done by maximizing the peak current while minimizing the transverse emittance. In three dimensions one must also minimize the beam energy spread and insure that the electron beam focusing is not so large that the Rayleigh length becomes short compared to the gain length.

At the APS it was our intent to capitalize on the existing infrastructure to construct an experimental beamline that was capable of testing the SASE process to saturation at wavelengths much shorter than had been achieved in the past [2]. As the key indicator of SASE is the exponential growth of the optical signal as a function of length along the undulator line, the experiment would be designed and instrumented so that it would be capable of measuring this exponential growth. Other key features would also be measured such as the mode size and divergence of the optical beam, the spectra, the electron beam bunching, and the nonlinear harmonics all as a function of distance along the undulator.

For initial simplicity we chose to begin operation at 530 $\mathrm{nm}$. This would allow us to perform some initial detailed measurements of the SASE process at an early time. Over time, as our electron beam properties improved, we would raise the energy of the electron beam and make measurements at shorter wavelengths. Table 1 shows the various phases of the project.

\section{EXPERIMENTAL SETUP}

The APS FEL system consists of the following elements: the photocathode rf gun and drive laser system; the APS linac; an electron bunch compressor; an
Table 1: Key Milestone Wavelengths and Necessary Beam Parameters for the APS FEL Project

\begin{tabular}{lccc}
\hline Wavelength [nm] & $\mathbf{5 3 0}$ & $\mathbf{1 2 0}$ & $\mathbf{5 9}$ \\
\hline Beam Energy [MeV] & 217 & 457 & 650 \\
Normalized Emittance [ $\mu \mathrm{m}]$ & 5 & 3 & 3 \\
Energy Spread [\%] & 0.1 & 0.1 & 0.1 \\
Peak Current [A] & 100 & 300 & 500 \\
Undulator Period [cm] & & 3.3 & \\
Undulator K Value & & 3.1 & \\
Gain Length [m] & 0.82 & 0.73 & 0.78 \\
Saturation Length [m] & 17.2 & 15.1 & 16.0 \\
Peak Power [MW] & 35 & 266 & 592 \\
\hline
\end{tabular}

undulator system; and various beam and optical diagnostics systems.

\subsection{Accelerator System}

The photocathode (PC) rf gun system is a copy of the BNL 1.6-cell S-band design [3,4]. The cathode material is copper. Electrons are generated at the proper rf phase by striking the cathode plane with a picosecond UV pulse from a high-power pulsed laser system. In order to insure proper arrival time of the light pulse relative to the $\mathrm{rf}$ phase, the laser oscillator is phased locked to the S-band drive frequency. Timing stability is roughly 1 degree at $2856 \mathrm{MHz}$.

We use the APS linac system to accelerate the electron pulse following generation within the PC rf gun system. The APS linac consists of thirteen 3-m S-band accelerating structures. Five 35-MW S-band klystrons are used to power the entire system. At full power and phased together the system is capable of accelerating the electron beam up to an energy of roughly $650 \mathrm{MeV}$.

In order to achieve high peak bunch currents, the electron bunch is compressed midway through the acceleration process. This is done by imparting a timecorrelated energy spread on the bunch via off-crest acceleration in the upstream $\mathrm{rf}$ cavities. The electron bunch is then passed through a magnetic chicane and the longitudinal phase space is rotated to produce a beam of high peak current. The increased energy spread is subsequently reduced by further acceleration of the beam.

There is a complete electron beam diagnostics suite along the length of the accelerator and transport lines. In particular it is possible to measure the energy spread, energy, Twiss parameters, and emittance at the bunch compressor. In addition to these properties we can also measure the electron bunch length at the end of the linac. Beam position monitors, fluorescent screens, and current monitors are located along the length of the accelerator and transport system.

\subsection{Undulator System}

The undulator system in use in the APS FEL system consists of individual cells. Within each cell is an undulator with a fixed gap of $8.3 \mathrm{~mm}$, a period of $3.3 \mathrm{~cm}$, and a $\mathrm{K}$ value of 3.1. The total length of a single undulator is $2.4 \mathrm{~m}$. 
A separate function undulator system is used. Individual undulators are spaced by roughly $38 \mathrm{~cm}$. Within the space is a horizontally focusing quadrupole and a diagnostics station. The natural focusing of the undulator is used to guide the beam in the vertical direction. We have had up to nine undulators installed in the line at one time.

\subsection{Primary Diagnostics System}

The primary diagnostics for the system are installed locally to its corresponding undulator. There are provisions to bring the FEL pulse out of the radiation enclosure to a laser-controlled area. At present there is only a high-resolution optical spectrometer installed at this external location.

Local electron beam diagnostics, those contained upand downstream of all undulators, consists of highresolution beam position monitors, optical transition radiation (OTR) and fluorescent YAG screens. With these we can measure and maintain the beam trajectory and size. The induced microbunching can also be observed by measuring the coherent OTR signal.

The suite of local optical diagnostics is similarly complete. We can measure the integrated optical intensity, the mode size and divergence, and spectra both before and after each undulator.

\section{MEASUREMENT AND ANALYSIS}

A measurement of the SASE properties is carried out by first making a complete measurement of the electron beam properties: Twiss parameter match, emittance, charge per bunch, bunch length, energy, and energy spread. As the SASE signal is also extremely sensitive to steering errors, it is used for fine tuning of the trajectory through the undulator system. Feedback systems are turned on to hold the beam properties constant, and the measurements of the optical signal are then made.

Table 2 lists the measured beam and optical properties for four sets of measurements. Data sets 1 and 2 contain the beam conditions and key measurements of the optical properties for both $530-\mathrm{nm}$ saturated and unsaturated conditions, while data set 3 contains similar information for a beam energy tuned to produce $385-\mathrm{nm}$ ultraviolet light. Data set 4 is again at $530 \mathrm{~nm}$, but with improved beam properties.

\subsection{Intensity vs. Distance}

Principle to proving that one is witnessing SASE is the measurement of the optical intensity as a function of distance. An exponentially growing signal should be measured. Due to the fact that SASE starts from noise, the SASE signal has large fluctuations shot-to-shot in intensity. This fluctuation is particularly acute during the exponential regime due both to the inherent fluctuations as well as shot-to-shot fluctuation in the beam properties and beam trajectory. It is important to measure the optical signal over many pulses and represent the output in a
Table 2: Beam and Optical Parameters for Four Different Conditions

\begin{tabular}{lcccc}
\hline Parameter & A & B & C & D \\
\hline Charge [nC] & 0.30 & 0.33 & 0.30 & 0.20 \\
rms Bunch Length [ps] & 0.19 & 0.77 & 0.65 & 0.30 \\
Peak Current [A] & 630 & 171 & 184 & 266 \\
Norm. Emittance [ $\mu \mathrm{m}]$ & 8.5 & 8.5 & 7.1 & 8.5 \\
Wavelength [nm] & 530 & 530 & 385 & 530 \\
Meas. Gain Length [m] & 0.97 & 1.4 & 0.76 & 0.59 \\
Calc. Gain Length [m] & 0.74 & 0.62 & 0.71 & 0.57 \\
\hline
\end{tabular}

proper statistical fashion. Furthermore, in order to make meaningful comparisons to simulation, the beam properties must be measured frequently. In all our measurements the electron beam properties are measured before the optical measurements. The optical measurements are then made at each of the diagnostics stations along the undulator system. The electron beam properties are then measured once again following the optical properties measurement in order to insure that no drift has occurred.

Figure 1A shows the integrated optical power as a function of distance for the beam conditions in column 1 of Table 2. Plotted are the 25, 50 (central dot), and 75 percentiles of the data set obtained at each diagnostics station. The vertical scale is in absolute units of energy.
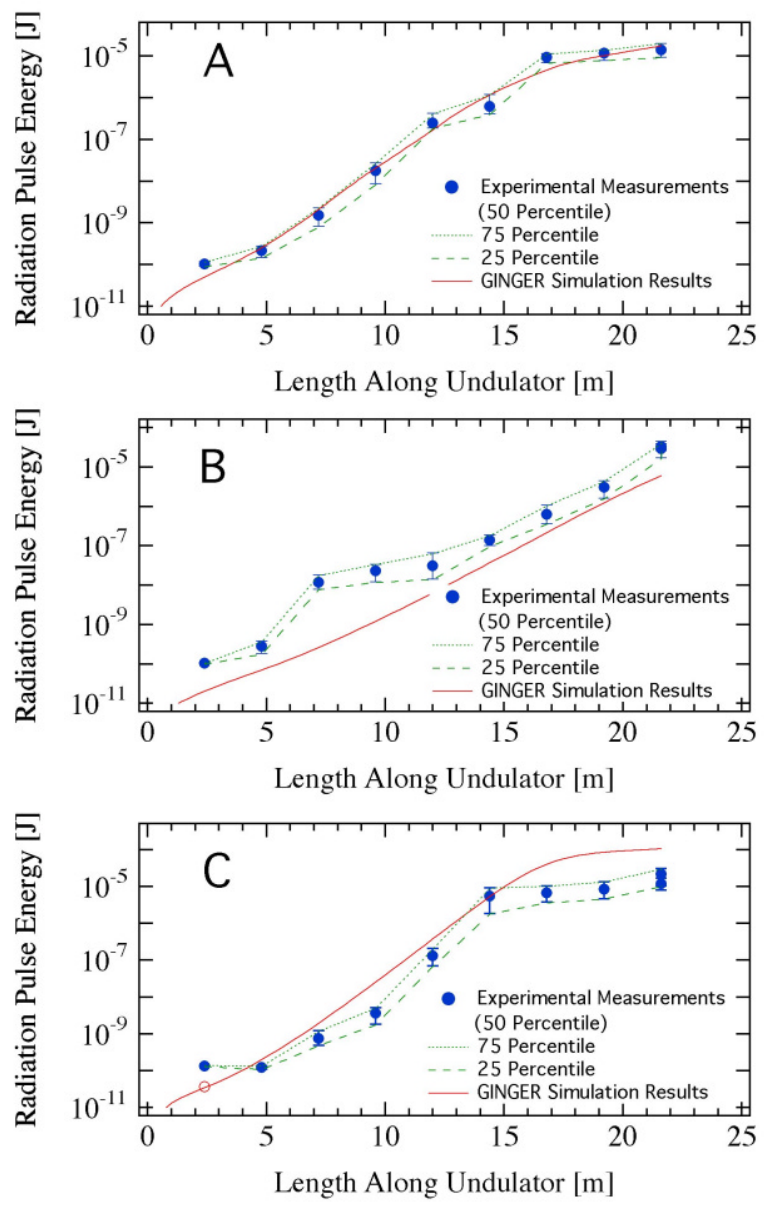

Figure 1: Pulse energy vs. distance: A) $530 \mathrm{~nm}$ saturated, B) $530 \mathrm{~nm}$ unsaturated, C) $385 \mathrm{~nm}$ saturated. 
The solid line is the simulation results from the SASE simulation code GINGER [5] averaged over 50 simulation runs using a statistical sample of input beams that mimic the measured beam parameters of Table 2. Again the absolute energy is plotted. The agreement is very good.

Figure $1 \mathrm{~B}$ is similar to Figure $1 \mathrm{~A}$ and is for the beam conditions of column 2 in Table 2. This time the beam was tuned so that saturation would not occur before the end of the undulator system showing that there is no problem with the last few undulators. Unfortunately the comparison to the simulation is not as good as in the previous case. The source of the discrepancy is not known.

Shorter wavelength is obtained by adjusting the beam energy. Data at $385 \mathrm{~nm}$ is shown in Figure 1C. This corresponds to the parameters found in column 3 Table 2 . Although the GINGER simulation agrees well in both the gain and saturation lengths, there is a discrepancy in the total energy achieved at saturation. We believe this is due to an effective bunch length shorter than what we had measured. This effect is still under investigation.

With further tuning and thus improvement in our ability to generate and maintain good electron beams came better SASE performance. Figure 2 shows a measurement of the intesity vs. distance for the parameter set displayed in column 4 of Table 2. Saturation of the signal is now clearly seen following the 5 th undulator (the $12-\mathrm{m}$ point).

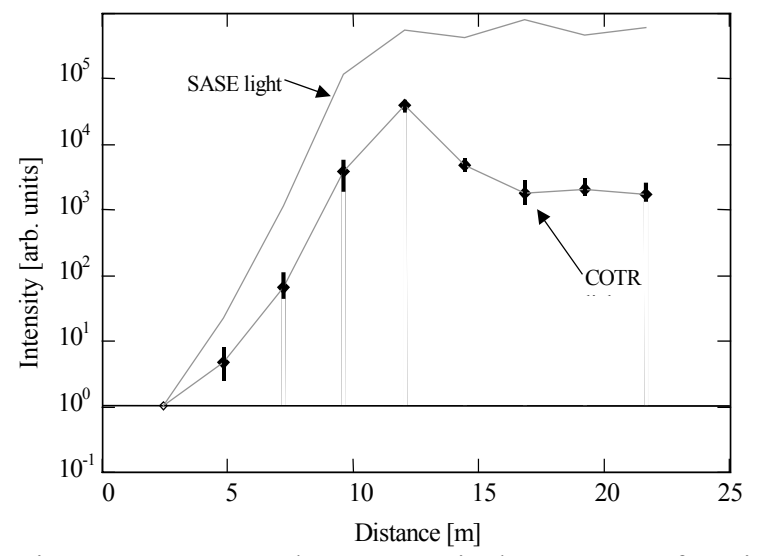

Figure 2: SASE and COTR optical power as a function of distance.

\subsection{Spectrum vs. Distance}

Measurement of the spectral properties as a function of distance reveals further features of the SASE process. As SASE develops, the longitudinal coherence length increases, and the naturally occurring temporal intensity spikes grow in width. This causes the bandwidth of the spectrum to narrow. One also observes a reduction in the number of measured frequency spikes. We measure the spectra as a function of distance along the undulator by deflecting the light out of the vacuum system and onto an optical spectrometer. The system is capable of single-shot measurements.

Figure 3 shows a representative measured spectrum as a function of distance along the undulator. VLD2 is near the beginning of the exponential growth, VLD5 is further on in the exponential growth, VLD7 is at the saturation point, and VLD9 is beyond saturation. One clearly sees the decrease in the bandwidth and the expected reduction in the number of spikes as one approaches saturation. Also shown is the simulation using similar beam parameters.

\subsection{Microbunching vs. Distance}

Complementary to the exponential growth of the optical signal is a microbunching of the electron beam at the resonant wavelength. This microbunching can be measured directly via observation of the coherent optical transition radiation (COTR) signal [6,7]. This signal is generated by the microbunched beam as it passes through a conductor.

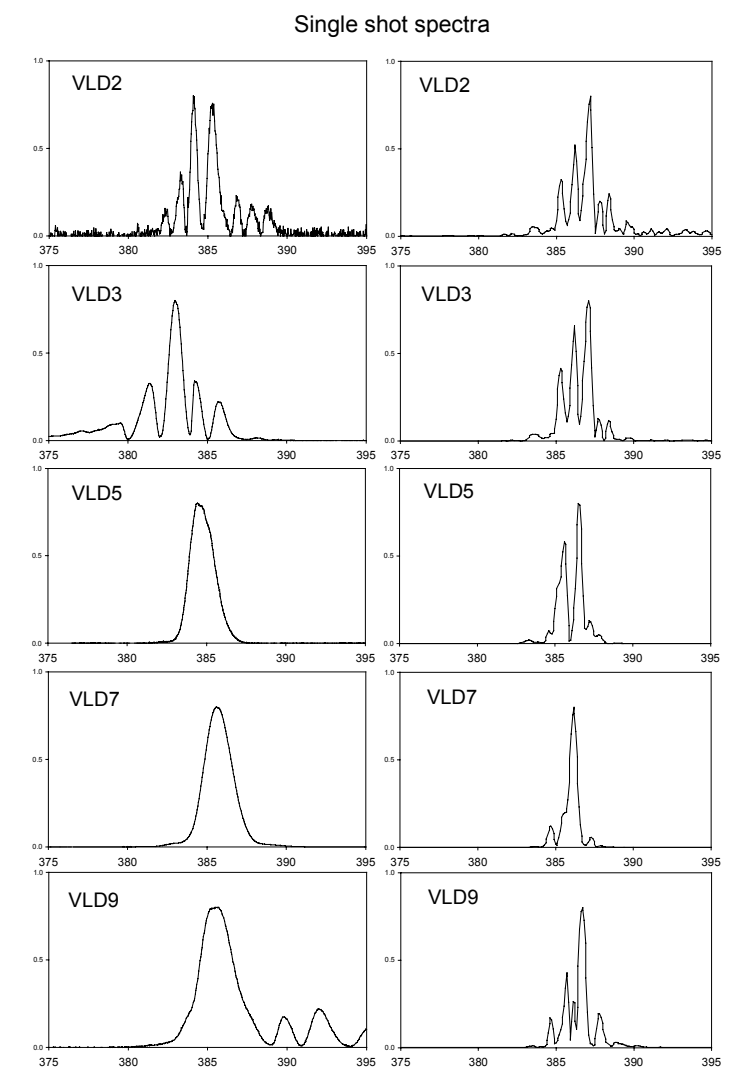

Figure 3: Z-dependent single-shot spectrum measurements of the SASE radiation (left column) and simulations (right column). The saturation is achieved around undulator 7 (VLD 7).

Measurements of the COTR signal were made at each diagnostics station by first inserting a thin foil to completely block the main optical signal. The beam passes through this thin foil unimpeded and goes on to strike a $45^{\circ}$ mirror that acts as the primary COTR radiator. The COTR signal is then measured using the same diagnostics system as used for the SASE signal. 
Growth of the COTR signal as a function of distance should also be exponential with a growth rate equal to that of the SASE signal. Figure 2 shows the COTR signal together with the SASE signal for the same beam parameter set. Following saturation the bunching decreases as observed by the drop in the COTR signal while the SASE signal remains roughly constant due to the optical pulse being confined in the vacuum chamber until extraction by the diagnostics mirrors of subsequent stations.

The spectrum of the COTR signal should also correspond to the signal generated by the optical pulse. It should be peaked at the resonant wavelength and have frequency spikes roughly equal to the number of developed coherence regions along the electron bunch. This spectrum is easily measured with the same diagnostics system as used for the SASE spectrum and indeed has a spectrum vary similar to that seen in Figure 3.

Further details of the experiment and a discussion of the results can be found in reference [8].

\section{SUMMARY}

We have measured the SASE signal from startup through to saturation of the process. Detailed measurements of the process have been made using many complementary signals. Simultaneous to the measurement of the SASE signal we have made careful measurements of the electron beam properties. These measurements have allowed us to make comparisons between the experimental results and the theoretical expectations. Our comparisons show good agreement, and we feel confident that the SASE process can be extended to much shorter wavelengths, possibly all the way to $x$-ray wavelengths.

We will continue to perform experiments on the SASE process at progressively shorter wavelengths. Further efforts will be focused on setting up a pioneering experiment to exploit the unique properties of the SASE light as well as testing more exotic ideas that could extend and enhance the capabilities of a SASE source.

\section{ACKNOWLEDGMENT}

This work is supported by the U.S. Department of Energy, Office of Basic Energy Sciences under Contract No. W-31-109-ENG-38.

\section{REFERENCES}

[1] See, for example, "Laser Handbook, Vol. 6, FreeElectron Lasers," ed. W. B. Colson, C. Pellegrini, and A. Renieri, North Holland (1990) and references therein.

[2] D.A. Kilpatrick, G. Bekefi, A.C. DiRienzo, H.P. Freund, A.K. Ganguly, Phys. Fluids B1, 1511 (1989).

[3] F. Sakai et al., "Development of High-Duty Operation RF Photoinjector," Report BNL-65003 (Brookhaven National Laboratory, Upton, NY, 1997).
[4] S. Biedron et al., in Proceedings of the IEEE 1999 Particle Accelerator Conference, A. Luccio, W. MacKay, Eds. (IEEE, Piscataway, NJ, 1999), pp. 2024-2026 (1999).

[5] W. Fawley, "An Informal Manual for GINGER and its post-processor XPLOTGIN," BP Tech Note-104 (Lawrence Berkeley National Laboratory, Berkeley, CA, 1995).

[6] A. Lumpkin et al., Phys. Rev. Lett. Vol. 86, No. 1, pp 79-81, (2001).

[7] A. Lumpkin et al., "First Observation of Microbunching Sidebands in a Saturated FEL Using Coherent Optical Transition Radiation," Phys. Rev. Lett., submitted July 2001.

[8] S. Milton et al., originally published in Science Express as $10.1126 /$ science.1059955 on May 17, 2001; Science, Vol. 292, Issue 5524, 2037-2041, June $15,2001$. 